Article

\title{
Oscillation of Emden-Fowler-Type Neutral Delay Differential Equations
}

\author{
Shyam Sundar Santra ${ }^{1, *,+}$, Taher A. Nofal ${ }^{2,3,+}$, Hammad Alotaibi ${ }^{2,+}$ and Omar Bazighifan ${ }^{4,5, *,+}$ (I) \\ 1 Department of Mathematics, JIS College of Engineering, Kalyani 741235, India \\ 2 Department of Mathematics, Faculty of Science, Taif University, Taif 21944, KSA, Saudi Arabia; \\ nofal_ta@yahoo.com (T.A.N.); h-85m@hotmail.com (H.A.) \\ 3 Mathematics Department, Faculty of Science, El- Minia University, El- Minia 61768, Egypt \\ 4 Department of Mathematics, Faculty of Science, Hadhramout University, Hadhramout 50512, Yemen \\ 5 Department of Mathematics, Faculty of Education, Seiyun University, Seiyun 50512, Yemen \\ * Correspondence: shyam01.math@gmail.com (S.S.S.); o.bazighifan@gmail.com (O.B.) \\ + These authors contributed equally to this work.
}

Received: 22 September 2020; Accepted: 5 November 2020; Published: 20 November 2020

\begin{abstract}
In this work, we consider a type of second-order functional differential equations and establish qualitative properties of their solutions. These new results complement and improve a number of results reported in the literature. Finally, we provide an example that illustrates our results.
\end{abstract}

Keywords: oscillation; non-oscillation; neutral; delay; Lebesgue's dominated convergence theorem

MSC: 34C10; 34K11

\section{Introduction}

Consider the class of Emden-Fowler-type neutral delay differential equations of the form:

$$
\left(f(y)\left(u^{\prime}(y)\right)^{c}\right)^{\prime}+h(y) w^{a}(\varsigma(y))=0 \quad \text { for } \quad y \geq y_{0}>0
$$

where $u(y)=w(y)+g(y) w(\vartheta(y))$ and $c$ and $a$ are the ratios of two odd positive integers. Let us consider the following conditions:

(a) $f, h, \vartheta, \varsigma \in C\left(\mathbb{R}_{+}, \mathbb{R}_{+}\right)$such that $\vartheta(y) \leq y, \varsigma(y) \leq y$ for $y \geq y_{0}, \vartheta(y) \rightarrow \infty \varsigma(y) \rightarrow \infty$ as $y \rightarrow \infty$;

(b) $\lim _{y \rightarrow \infty} \Lambda(y)=\infty$ where $\Lambda(y)=\int_{0}^{y}(f(\eta))^{-1 / c} d \eta$;

(c) $g \in C\left(\mathbb{R}_{+}, \mathbb{R}_{-}\right)$with $-1+(2 / 5)^{1 / c} \leq-a \leq g(y) \leq 0$ for $y \in \mathbb{R}_{+}$;

(d) $g \in C\left(\mathbb{R}_{+}, \mathbb{R}_{-}\right)$with $-1<-a \leq g(y) \leq 0$ for $y \in \mathbb{R}_{+}$.

Brands [1] showed that for bounded delays, the solutions to:

$$
w^{\prime \prime}(y)+h(y) w(y-\vartheta(y))=0
$$

are oscillatory, if and only if the solutions to $w^{\prime \prime}(y)+h(y) w(y)=0$ are oscillatory. Baculikova and Džurina [2] studied (1) when $c=a=1$, by considering the assumptions $0 \leq g(y)<\infty$ and $\lim _{y \rightarrow \infty} \Lambda(y)=\infty$. They obtained sufficient conditions for the oscillation of the solutions of (1), using comparison techniques. In another paper, Baculikova and Džurina [3] considered (1) and established sufficient conditions for the oscillation of the solutions of (1) under the assumptions $0 \leq g(y)<\infty$ and $\lim _{y \rightarrow \infty} \Lambda(y)=\infty$. Bohner et al. [4] established sufficient conditions for the oscillation of the solutions of (1) when $c=a$ and assuming $\lim _{y \rightarrow \infty} \Lambda(y)<\infty$ and $0 \leq g(y)<1$. 
Chatzarakis et al. [5] established sufficient conditions for the oscillation and asymptotic behavior of all solutions of second-order half-linear differential equations of the form:

$$
\left(f(y)\left(w^{\prime}(y)\right)^{a}\right)^{\prime}+h(y) w^{a}(\vartheta(y))=0
$$

In subsequent work, Chatzarakis et al. [6] established improved oscillation criteria for (2). Džurina [7] established sufficient conditions for the oscillation of the solutions of (1) when $c=a=1$, by considering the assumptions $\lim _{y \rightarrow \infty} \Lambda(y)=\infty$ and $0 \leq g(y) \leq g_{0}<\infty$. Fisnarova and Marik [8] considered the equation:

$$
\left(f(y) \Phi\left(u^{\prime}(y)\right)\right)^{\prime}+c(y) \Phi(w(s(y)))=0,
$$

where $u(y)=w(y)+g(y) w(\vartheta(y))$ and $\Phi(y)=|y|^{p-2} y, p \geq 2$. Umar et al. [9] studied stochastic intelligent computing with neuro-evolution heuristics for nonlinear SITRsystem of novel COVID-19 dynamics. Guirao et al. [10] considered novel third-order nonlinear Emden-Fowler delay differential equations, designed a model, as well as found the numerical solutions of third-order nonlinear Emden-Fowler delay differential equations. Touchent et al. [11] established the modified invariant subspace method for solving partial differential equations with non-singular kernel fractional derivatives. Sabir et al. [12] considered second-order singular periodic nonlinear boundary value problems and performed the computing based on neuro-swarm intelligence. Grace et al. [13] established sufficient conditions for the oscillation of the solutions of (1) when $c=a$ and by considering the assumptions $\lim _{y \rightarrow \infty} \Lambda(y)<\infty, \lim _{y \rightarrow \infty} \Lambda(y)=\infty$, and $0 \leq g(y)<1$. Karpuz and Santra [14] obtained several sufficient conditions for the oscillatory and asymptotic behavior of the solutions of:

$$
\left(f(y)(w(y)+g(y) w(\vartheta(y)))^{\prime}\right)^{\prime}+h(y) f(w(\varsigma(y)))=0
$$

by considering the assumptions $\lim _{y \rightarrow \infty} \Lambda(y)<\infty$ and $\lim _{y \rightarrow \infty} \Lambda(y)=\infty$, for different ranges of $g$. Li et al. [15] established sufficient conditions for the oscillation of the solutions of (1), under the assumptions $\lim _{y \rightarrow \infty} \Lambda(y)<\infty$ and $g(y) \geq 0$.

For further work on the oscillation of the solutions to this type of equation, we refer the readers to [16-27]. Note that the majority of publications consider only sufficient conditions, and merely a few consider necessary and sufficient conditions. Hence, the objective in this work is to establish both necessary and sufficient conditions for the oscillatory and asymptotic behavior of the solutions of (1) without using the comparison and the Riccati techniques.

Many researchers investigated regularity properties and obtained several existence results of solutions to partial differential equations; see [28-30] and the references therein. Furthermore, the authors in $[31,32]$ considered the study of the exact and approximate solutions to differential equations.

Neutral differential equations have several applications in the natural sciences and engineering. For example, they often appear in the study of distributed networks containing lossless transmission lines (see, e.g., [33]). In this paper, we restrict our attention to the study of (1), which includes the class of functional differential equations of the neutral type.

By a solution to Equation (1), we mean a function $w \in C\left(\left[Y_{w}, \infty\right), \mathbb{R}\right)$, where $Y_{w} \geq y_{0}$, such that $f u^{\prime} \in C^{1}\left(\left[Y_{w}, \infty\right), \mathbb{R}\right)$, satisfying (1) on the interval $\left[Y_{w}, \infty\right)$. A solution $w$ of $(1)$ is said to be proper if $w$ is not identically zero eventually, i.e., $\sup \{|w(y)|: y \geq Y>0\}>0$ for all $Y \geq Y_{w}$. We assume that (1) possesses such solutions. A solution of (1) is called oscillatory if it has arbitrarily large zeros on $\left[Y_{w}, \infty\right)$; otherwise, it is said to be non-oscillatory. (1) itself is said to be oscillatory if all of its solutions are oscillatory.

Remark 1. When the domain is not specified explicitly, all functional inequalities considered in this paper are assumed to hold eventually, i.e., they are satisfied for all $y$ large enough. 


\section{Main Results}

Lemma 1. Let $(a),(b)$, and one of $(c)$ or $(d)$ hold. Furthermore, let $w$ be an eventually positive solution of (1). Then, $u$ satisfies one of the following two possible cases:

(i) $\quad u(y)<0, \quad u^{\prime}(y)>0$ and $\left(f(y)\left(u^{\prime}(y)\right)^{c}\right)^{\prime}<0$;

(ii) $u(y)>0, \quad u^{\prime}(y)>0$ and $\quad\left(f(y)\left(u^{\prime}(y)\right)^{c}\right)^{\prime}<0$

for $y_{2} \geq y_{1}>0$, where $y \geq y_{2}$ is sufficiently large.

Proof. Suppose that there exists a $y_{1} \geq Y_{w}$ such that $w(y)>0, w(\vartheta(y))$, and $w(\varsigma(y))>0$ for $y \geq y_{1}$. From (1) and (a), we have that:

$$
\left(f(y)\left(u^{\prime}(y)\right)^{c}\right)^{\prime}=-h(y) w^{a}(s(y))<0 \quad \text { for } y \geq y_{1}
$$

Consequently, $\left(f(y)\left(u^{\prime}(y)\right)^{c}\right)$ is nonincreasing on $\left[y_{1}, \infty\right)$. Since $f(y)>0$, thus either $u^{\prime}(y)<0$ or $u^{\prime}(y)>0$ for $y \geq y_{2}$, where $y_{2} \geq y_{1}$.

If $u^{\prime}(y)>0$ for $y \geq y_{2}$, then we have (i) and (ii). We prove now that $u^{\prime}(y)<0$ cannot occur.

If $u^{\prime}(y)<0$ for $y \geq y_{2}$, then there exists $\kappa>0$ such that $\left(f(y)\left(u^{\prime}(y)\right)^{c}\right) \leq-\kappa$ for $y \geq y_{2}$. Integrating over $\left[y_{2}, y\right) \subset\left[y_{2}, \infty\right)$ after dividing by $f$, we have:

$$
u(y) \leq u\left(y_{2}\right)-\kappa^{1 / c} \int_{y_{2}}^{y}(f(\eta))^{-1 / c} d \eta \quad \text { for } y \geq y_{2}
$$

By virtue of Condition $(b), \lim _{y \rightarrow \infty} u(y)=-\infty$. We consider now the following possible cases, separately.

If $w$ is unbounded, then there exists a sequence $\left\{y_{k}\right\}$ such that $\lim _{k \rightarrow \infty} y_{k}=\infty$ and $\lim _{k \rightarrow \infty} w\left(y_{k}\right)=\infty$, where $w\left(y_{k}\right)=\max \left\{w(\eta) ; y_{0} \leq \eta \leq y_{k}\right\}$. Since $\lim _{y \rightarrow \infty} \vartheta(y)=\infty, \vartheta\left(y_{k}\right)>y_{0}$ for all sufficiently large $k$. By $\vartheta(y) \leq y$,

$$
w\left(\vartheta\left(y_{k}\right)\right)=\max \left\{w(\eta) ; y_{0} \leq \eta \leq \vartheta\left(y_{k}\right)\right\} \leq \max \left\{w(\eta) ; y_{0} \leq \eta \leq y_{k}\right\}=w\left(y_{k}\right) .
$$

Therefore, for all large $k$,

$$
u\left(y_{k}\right)=w\left(y_{k}\right)+g\left(y_{k}\right) w\left(\vartheta\left(y_{k}\right)\right) \geq\left(1+g\left(y_{k}\right)\right) w\left(y_{k}\right)>0,
$$

which contradicts the fact that $\lim _{y \rightarrow \infty} u(y)=-\infty$.

If $w$ is bounded, then $u$ is also bounded, which contradicts $\lim _{y \rightarrow \infty} u(y)=-\infty$. Hence, $u$ satisfies one of the cases (i) and (ii). This completes the proof.

Lemma 2. Let $(a),(b)$, and one of $(c)$ or $(d)$ hold. Furthermore, let $w$ be an eventually positive unbounded solution of (1). Then, $u$ satisfies (ii), only.

Proof. Suppose that there exists a $y_{1} \geq y_{0}$ such that $w(y)>0, w(\vartheta(y))$, and $w(\varsigma(y))>0$ for $y \geq y_{1}$. Then, Lemma 1 holds, and $u$ satisfies (i) and (ii) for $y_{2} \geq y_{1}$, where $y \geq y_{2}$. By Lemma 1 , if $w$ is unbounded, then $u(y)>0$. Therefore, (i) cannot occur. This completes the proof.

Lemma 3. Let $(a),(b)$, and one of $(c)$ or $(d)$ hold. Furthermore, let $w$ be an eventually positive solution of (1) and $u$ satisfy (i). Then, $\lim _{y \rightarrow \infty} w(y)=0$. 
Proof. Suppose that there exists $y_{1} \geq y_{0}$ such that $w(y)>0, w(\vartheta(y))$, and $w(\varsigma(y))>0$, for $y \geq y_{1}$. Then, Lemma 1 holds, and $u$ satisfies (i) and (ii) for $y_{2} \geq y_{1}$, where $y \geq y_{2}$. Let $u$ satisfy (i) for $y \geq y_{2}$. Therefore,

$$
\begin{aligned}
0 \geq \lim _{y \rightarrow \infty} u(y) & =\limsup _{y \rightarrow \infty} u(y) \geq \limsup _{y \rightarrow \infty}(w(y)-a x(\vartheta(y))) \\
& \geq \limsup _{y \rightarrow \infty} w(y)+\liminf _{y \rightarrow \infty}(-a w(\vartheta(y)))=(1-a) \limsup _{y \rightarrow \infty} w(y)
\end{aligned}
$$

which implies that $\limsup _{y \rightarrow \infty} w(y)=0$, and hence, $\lim _{y \rightarrow \infty} w(y)=0$.

\subsection{The Case $c>a$}

In this subsection, we assume that there exists a constant $b>0$ such that $0<a<b<c$.

Lemma 4. Let Assumptions (a), (b), (c), or (d) be satisfied. Furthermore, assume $w$ is an eventually positive solution of (1) and $u$ satisfies (ii). Then, there exists $y_{2} \geq y_{1}$ and $\kappa>0$ such that for $y \geq y_{2}$, the following inequalities hold:

$$
\begin{gathered}
u(y) \leq \kappa^{1 / c} \Lambda(y) \\
\left(\Lambda(y)-\Lambda\left(y_{1}\right)\right)\left[\int_{y}^{\infty} h(\zeta)\left(\kappa^{1 / c} \Lambda(\varsigma(\zeta))\right)^{a-b} u^{b}(\varsigma(\zeta)) d \zeta\right]^{1 / c} \leq u(y) .
\end{gathered}
$$

Proof. Suppose that there exists $y_{1} \geq y_{0}$ such that $w(y)>0, w(\vartheta(y))$, and $w(\varsigma(y))>0$ for $y \geq y_{1}$. Then, Lemma 1 holds, and $u$ satisfies (i) and (ii) for $y_{2} \geq y_{1}$, where $y \geq y_{2}$. Let $u$ satisfy (ii) for $y \geq y_{2}$. By (ii), $\left(f(y)\left(u^{\prime}(y)\right)^{c}\right)$ is positive and non-increasing. Therefore, there exists $\kappa>0$ and $y_{2} \geq y_{1}$ such that $\left(f(y)\left(u^{\prime}(y)\right)^{c}\right) \leq \kappa$. Integrating the inequality $u^{\prime}(y) \leq(\kappa / f(y))^{1 / c}$, we have:

$$
u(y) \leq u\left(y_{2}\right)+\kappa^{1 / c}\left(\Lambda(y)-\Lambda\left(y_{2}\right)\right)
$$

Since $\lim _{y \rightarrow \infty} \Lambda(y)=\infty$, the last inequality becomes:

$$
u(y) \leq \kappa^{1 / c} \Lambda(y) \text { for } y \geq y_{2}
$$

which is (5). Note that $\kappa$ depends on $w$ being evaluated at a time $y_{2}$. Thus, (6) must include all possible $\kappa^{\prime}$ s.

From (5), we have:

$$
u^{a}(\varsigma(y))=u^{a-b}(\varsigma(y)) u^{b}(\varsigma(y)) \geq\left(\kappa^{1 / c} \Lambda(\varsigma(y))\right)^{a-b} u^{b}(\varsigma(y))
$$

Using $u(y) \leq w(y)$ and (7) in (1) and then integrating the final inequality from $y$ to $\infty$, we have:

$$
\lim _{A \rightarrow \infty}\left[\left(f(\eta)\left(u^{\prime}(\eta)\right)^{c}\right)\right]_{y}^{A}+\int_{y}^{\infty} h(\eta)\left(\kappa^{1 / c} \Lambda(\varsigma(\eta))\right)^{a-b} u^{b}(\varsigma(\eta)) d \eta \leq 0 .
$$

Using that $\left(f(y)\left(u^{\prime}(y)\right)^{c}\right)$ is positive and non-increasing, we have:

$$
\int_{y}^{\infty} h(\eta)\left(\kappa^{1 / c} \Lambda(\zeta(\eta))\right)^{a-b} u^{b}(\varsigma(\eta)) d \eta \leq\left(f(y)\left(u^{\prime}(y)\right)^{c}\right) \quad \text { for } y \geq y_{1} \text {. }
$$

Therefore,

$$
u^{\prime}(y) \geq\left[\frac{1}{f(y)} \int_{y}^{\infty} h(\eta)\left(\kappa^{1 / c} \Lambda(\zeta(\eta))\right)^{a-b} u^{b}(\zeta(\eta)) d \eta\right]^{1 / c}
$$


Since $u(y) \geq 0$, integrating (8) from $y_{2}$ to $y$, we obtain:

$$
\begin{aligned}
u(y) & \geq \int_{y_{2}}^{y}\left[\frac{1}{f(\eta)} \int_{\eta}^{\infty} h(\zeta)\left(\kappa^{1 / c} \Lambda(\zeta(\zeta))\right)^{a-b} u^{b}(\zeta(\zeta)) d \zeta\right]^{1 / c} d \eta \\
& \geq\left(\Lambda(y)-\Lambda\left(y_{2}\right)\right)\left[\int_{y}^{\infty} h(\zeta)\left(\kappa^{1 / c} \Lambda(\varsigma(\zeta))\right)^{a-b} u^{b}(\zeta(\zeta)) d \zeta\right]^{1 / c}
\end{aligned}
$$

which is (6).

Theorem 1. Under Assumptions (a)-(c), every unbounded solution of (1) oscillates if and only if:

(e) $\int_{T}^{\infty} h(\eta) \Lambda^{a}(\varsigma(\eta)) d \eta=+\infty$ for every $T>0$.

Proof. To prove sufficiency by contradiction, assume that $w$ is a non-oscillatory unbounded solution of (1). Then, there exists $y_{1} \geq y_{0}$ such that either $w(y)>0$ or $w(y)<0$ for $y \geq y_{1}$. Assume that $w(y)>0, w(\vartheta(y))>0$ and $w(\varsigma(y))>0$ for $y \geq y_{1}$. Then, Lemmas 1,2 , and 4 hold for $y \geq y_{2}$. From Lemma 1, $u$ satisfies (i) and (ii) for $y \geq y_{2}$. Again, from Lemma 2, $u$ satisfies (ii) only for $y \geq y_{2}$. Therefore, by Lemma 4, we have:

$$
u(y)>\left(\Lambda(y)-\Lambda\left(y_{1}\right)\right) \triangle^{1 / c}(y) \text { for all } y \geq y_{2},
$$

where:

$$
\triangle(y)=\int_{y}^{\infty} h(\zeta)\left(\kappa^{1 / c} \Lambda(\varsigma(\zeta))\right)^{a-b} u^{b}(\varsigma(\zeta)) d \zeta \geq 0
$$

Since $\lim _{y \rightarrow \infty} \Lambda(y)=\infty$, there exists $y_{3} \geq y_{2}$, such that $\Lambda(y)-\Lambda\left(y_{1}\right) \geq \frac{1}{2} \Lambda(y)$ for $y \geq y_{3}$. Then:

$$
u(y)>\frac{1}{2} \Lambda(y) \triangle^{1 / c}(y) \text { for } y \geq y_{3},
$$

and $u^{b} /\left(\kappa^{1 / c} \Lambda\right)^{b} \geq \triangle^{b / c} /\left(2 \kappa^{1 / c}\right)^{b}$. Taking the derivative of $\triangle$, we have:

$$
\begin{aligned}
\triangle^{\prime}(y) & =-h(y)\left(\kappa^{1 / c} \Lambda(\varsigma(y))\right)^{a-b} u^{b}(\varsigma(y)) \\
& \leq-h(y)\left(\kappa^{1 / c} \Lambda(\varsigma(y))\right)^{a} \triangle^{b / c}(\varsigma(y))\left(2 \kappa^{1 / c}\right)^{-b} \leq 0 .
\end{aligned}
$$

Therefore, $\triangle(y)$ is non-increasing so $\triangle^{b / c}(\zeta(y)) / \triangle^{b / c}(y) \geq 1$, and:

$$
\left(\triangle^{1-b / c}(y)\right)^{\prime}=(1-b / c) \triangle^{-b / c}(y) \triangle^{\prime}(y) \leq-(1-b / c) 2^{-b} \kappa^{(a-b) / c} h(y) \Lambda^{a}(\varsigma(y)) .
$$

Integrating this inequality from $y_{3}$ to $y$, we have:

$$
\left[\triangle^{1-b / c}(\eta)\right]_{y_{3}}^{y} \leq-(1-b / c) 2^{-b} \kappa^{(a-b) / c} \int_{y_{3}}^{y} h(\eta) \Lambda^{a}(\varsigma(\eta)) d \eta .
$$

Since $b / c<1$ and $\triangle(y)$ is positive and non-increasing, we have:

$$
\int_{y_{3}}^{y} h(\eta) \Lambda^{a}(\varsigma(\eta)) d \eta \leq \frac{2^{b} \kappa^{(b-a) / c}}{(1-b / c)} \triangle^{1-b / c}\left(y_{3}\right) .
$$

This contradicts (e) and proves the oscillation of all solutions.

If $w(y)<0$ for $y \geq y_{1}$, then we set $x(y):=-w(y)$ for $y \geq y_{1}$ in (1). Therefore,

$$
\left(f\left(\bar{u}^{\prime}\right)^{c}\right)(y)+h(y) x^{a}(\varsigma(y))=0 \quad \text { for } y \geq y_{1},
$$


where $\bar{u}(y)=x(y)+g(y) x(\vartheta(y))$. Then, proceeding as above, we reach the same contradiction. This proves the oscillation of all unbounded solutions of (1).

Next, we show that (e) is a necessary condition. Suppose that (e) does not hold; so, for some $\kappa>0$, the integral in (e) is finite. Therefore, there exists $y \geq y_{0}$ such that:

$$
\int_{Y}^{\infty} h(\eta) \Lambda^{a}(\varsigma(\eta)) d \eta \leq \frac{\kappa^{1-a / c}}{5} .
$$

Let us consider the closed subset $M$ of continuous functions:

$$
\begin{gathered}
M=\left\{w: w \in C\left(\left[y_{0}, \infty\right), \mathbb{R}\right), w(y)=0 \text { for } y \in\left(y_{0}, Y\right]\right. \text { and } \\
\left.\left(\frac{\kappa}{5}\right)^{1 / c}[\Lambda(y)-\Lambda(y)] \leq w(y) \leq \kappa^{1 / c}[\Lambda(y)-\Lambda(y)] \text { for } y \geq y_{0}\right\}
\end{gathered}
$$

We define the operator $\Phi: M \rightarrow C\left(\left[y_{0},+\infty\right), \mathbb{R}\right)$ by:

$$
(\Phi w)(y)=\left\{\begin{array}{l}
0, \quad y \in\left(y_{0}, Y\right] \\
-g(y) w(\vartheta(y))+\int_{Y}^{y}\left[\frac{1}{f(\eta)}\left[\frac{\kappa}{5}+\int_{\eta}^{\infty} h(\zeta) w^{a}(\varsigma(\zeta)) d \zeta\right]\right]^{1 / c} d \eta, y \geq Y .
\end{array}\right.
$$

For every $w \in M$ and $y \geq Y$, we have:

$$
\begin{aligned}
(\Phi w)(y) & \geq \int_{Y}^{y}\left[\frac{1}{f(\eta)}\left[\frac{\kappa}{5}+\int_{\eta}^{\infty} h(\zeta) w^{a}(\varsigma(\zeta)) d \zeta\right]\right]^{1 / c} d \eta \\
& \geq \int_{Y}^{y}\left[\frac{1}{f(\eta)} \frac{\kappa}{5}\right]^{1 / c} d \eta=\left(\frac{\kappa}{5}\right)^{1 / c}[\Lambda(y)-\Lambda(y)]
\end{aligned}
$$

For every $w \in M$ and $y \geq Y$, we have $w(y) \leq \kappa^{1 / c} \Lambda(y)$ and $w^{a}(y) \leq \kappa^{a / c} \Lambda^{a}(y)$. Then:

$$
\begin{aligned}
(\Phi w)(y) & \leq-g(y) w(\vartheta(y))+\int_{Y}^{y}\left[\frac{1}{f(\eta)}\left(\frac{\kappa}{5}+\frac{\kappa}{5}\right)\right]^{1 / c} d \eta \\
& \leq a \kappa^{1 / c}[\Lambda(\vartheta(y))-\Lambda(y)]+(2 \kappa / 5)^{1 / c}[\Lambda(y)-\Lambda(y)] \\
& \leq a \kappa^{1 / c}[\Lambda(y)-\Lambda(y)]+(2 \kappa / 5)^{1 / c}[\Lambda(y)-\Lambda(y)] \\
& =\left(a+(2 / 5)^{1 / c}\right) \kappa^{1 / c}[\Lambda(y)-\Lambda(y)] \leq \kappa^{1 / c}[\Lambda(y)-\Lambda(y)]
\end{aligned}
$$

which implies that $(\Phi w)(y) \in M$. Let us define now a sequence of continuous function $v_{n}:\left[y_{0},+\infty\right) \rightarrow$ $\mathbb{R}$ by the recursive formula:

$$
\begin{gathered}
v_{0}(y)= \begin{cases}0, & y \in\left(y_{0}, Y\right] \\
\frac{\kappa}{5}[\Lambda(y)-\Lambda(y)], & y \geq Y .\end{cases} \\
v_{n}(y)=\left(\Phi v_{n-1}\right)(y), \quad n \geq 1
\end{gathered}
$$

It is easy to verify that for $n>1$,

$$
\left(\frac{\kappa}{5}\right)^{1 / c}[\Lambda(y)-\Lambda(y)] \leq u_{n-1}(y) \leq u_{n}(y) \leq \kappa^{1 / c}[\Lambda(y)-\Lambda(y)]
$$

Therefore, the pointwise limit of the sequence exists. Let $\lim _{y \rightarrow \infty} v_{n}(y)=v(y)$ for $y \geq y_{0}$. By Lebesgue's dominated convergence theorem, $v \in M$ and $(\Phi v)(y)=v(y)$, where $v(y)$ is a solution of $(1)$ on $[T, \infty)$ such that $v(y)>0$. Hence, (e) is necessary. This completes the proof. 
Example 1. Consider the delay differential equation:

$$
\left(e^{-y}\left(\left(w(y)-e^{-y} w(y-1)\right)^{\prime}\right)^{3 / 5}\right)^{\prime}+y(w(y-2))^{1 / 3}=0, \quad y \geq 0 .
$$

Here, $c=3 / 5, a=1 / 3, f(y)=e^{-y},-1<g(y)=-e^{-y} \leq 0, \vartheta(y)=y-1, \varsigma(y)=y-2$, $\Lambda(y)=\int_{0}^{y} e^{5 \eta / 3} d \eta=\frac{3}{5}\left(e^{5 y / 3}-1\right)$. For $b=1 / 2$ and $p \leq q$, we have $0<a<b<c$ and $p^{a-b}=p^{-1 / 6} \geq$ $q^{a-b}=q^{-1 / 6}$. To check (e), we have:

$$
\int_{0}^{\infty} h(\eta) \Lambda^{a}(\zeta(\eta)) d \eta=\int_{0}^{\infty} \eta\left(\frac{3}{5}\left(e^{5(\eta-2) / 3}-1\right)\right)^{1 / 3} d \eta=\infty,
$$

since the integral approaches $+\infty$ as $\eta \rightarrow+\infty$. Therefore, all the assumptions of Theorem 1 hold. Thus, every unbounded solution of (9) oscillates.

Theorem 2. Under Assumptions (a)-(c), every solution of (1) oscillates or $\lim _{y \rightarrow \infty} w(y)=0$ if and only if (e) holds.

Proof. We show sufficiency by contradiction. Assume that $w$ is an eventually positive solution of (1). Then, there exists $y_{1} \geq y_{0}$ such that $w(y)>0, w(\vartheta(y))>0$, and $w(\varsigma(y))>0$ for $y \geq y_{1}$. Then, Lemmas 1, 3, and 4 hold for $y \geq y_{2}$. From Lemma $1, u$ satisfies (i) and (ii) for $y \geq y_{2}$.

If $u$ satisfies (i), then $\lim _{y \rightarrow \infty} w(y)=0$ due to Lemma 3 .

If $u$ satisfies (ii), then we proceed as in Theorem 1 to get a contradiction to (e). Thus, (e) is a sufficient condition.

The case where $w$ is a negative solution is similar, and we omit it here.

The necessary part is the same as in Theorem 1 . Thus, the proof of the Theorem is complete.

\subsection{The Case $c<a$}

In this subsection, we assume that there exists a constant $b>0$ such that $a>b>c>0$.

Lemma 5. Assume (a), (b), and one of (c) or (d) are satisfied. Let $w$ be an eventually positive solution of (1) and $u$ satisfy (ii). Then, there exists $y_{2} \geq y_{1}$ and $\kappa>0$ such that for $y \geq y_{2}$, the following inequality holds:

$$
\left.u^{a}(\varsigma(y))\right) \geq \kappa^{a-b} u^{b}(\varsigma(y)) .
$$

Proof. Suppose that there exists $y_{1} \geq y_{0}$ such that $w(y)>0, w(\vartheta(y))$, and $w(\varsigma(y))>0$ for $y \geq y_{1}$. Then, Lemma 1 holds, and $u$ satisfies (i) and (ii) for $y_{2} \geq y_{1}$, where $y \geq y_{2}$. Let $u$ satisfy (ii) for $y \geq y_{2}$. By (ii), it follows that $u^{\prime}(y)>0$, so $u$ is increasing, and $u(y) \geq u\left(y_{2}\right)$ for $y \geq y_{2}$. Thus:

$$
u(\varsigma(y)) \geq u\left(\varsigma\left(y_{2}\right)\right):=\kappa>0 \text { for } y \geq y_{2}:=y_{1} .
$$

Therefore,

$$
u^{a}(\varsigma(y))=u^{a-b}(\varsigma(y)) u^{b}(\varsigma(y)) \geq \kappa^{a-b} u^{b}(\varsigma(y)) \text { for } y \geq y_{2}
$$

which is (10).

Theorem 3. Assume that (a), (b), and (d) hold. Furthermore, assume that $\varsigma^{\prime}(y)>1$ and $r^{\prime}(y) \geq 0$ for $y \in \mathbb{R}_{+}$. Then, every solution of (1) oscillates or $\lim _{y \rightarrow \infty} w(y)=0$ if and only if:

$$
\text { (f) } \int_{T}^{\infty}\left[\frac{1}{f(\eta)}\left[\int_{\eta}^{\infty} h(\zeta) d \zeta\right]\right]^{1 / c} d \eta=+\infty \quad \text { for every } \quad T>0 \text {. }
$$


Proof. We show sufficiency by contradiction. Assume that $w$ is an eventually positive solution of (1). Then, there exists $y_{1} \geq y_{0}$ such that $w(y)>0, w(\vartheta(y))>0$, and $w(\varsigma(y))>0$ for $y \geq y_{1}$. From Lemma $1, u$ satisfies (i) and (ii) for $y \geq y_{2}$.

If $u$ satisfies (i), then $\lim _{y \rightarrow \infty} w(y)=0$ due to Lemma 3 .

If $u$ satisfies (ii), then Lemma 5 holds for $y \geq y_{2}$. Using $u(y) \leq w(y)$ and (10) in (1) and integrating the final inequality from $y$ to $\infty$, we have:

$$
\lim _{A \rightarrow \infty}\left[\left(f(\eta)\left(u^{\prime}(\eta)\right)^{c}\right)^{\prime}\right]_{y}^{A}+\kappa^{a-b} \int_{y}^{\infty} h(\eta) u^{b}(\varsigma(\eta)) d \eta \leq 0 .
$$

Using that $\left(f(y)\left(w^{\prime}(y)\right)^{c}\right)$ is positive and non-increasing and $r^{\prime}(y) \geq 0$, we have:

$$
\kappa^{a-b} \int_{y}^{\infty} h(\eta) u^{b}(\varsigma(\eta)) d \eta \leq\left(f(y)\left(u^{\prime}(y)\right)^{c}\right) \leq\left(f(\varsigma(y))\left(u^{\prime}(\varsigma(y))\right)^{c}\right) \leq f(y)\left(u^{\prime}(\varsigma(y))\right)^{c}
$$

for all $y \geq y_{2}$. Therefore,

$$
u^{\prime}(\varsigma(y)) \geq \kappa^{(a-b) / c}\left[\frac{1}{f(y)} \int_{y}^{\infty} h(\eta) u^{b}(\varsigma(\eta)) d \eta\right]^{1 / c} \geq \kappa^{(a-b) / c} u^{b / c}(\varsigma(y))\left[\frac{1}{f(y)} \int_{y}^{\infty} h(\eta) d \eta\right]^{1 / c}
$$

implies that:

$$
\kappa^{(a-b) / c}\left[\frac{1}{f(y)} \int_{y}^{\infty} h(\eta) d \eta\right]^{1 / c} \leq \frac{u^{\prime}(\varsigma(y))}{u^{b / c}(\varsigma(y))}
$$

Since $b / c>1$ and $u^{\prime}(\varsigma(y)) \leq u^{\prime}(\varsigma(y)) \varsigma^{\prime}(y)$, integrating (11) from $y_{2}$ to $\infty$, we have:

$$
\kappa^{(a-b) / c} \int_{y_{2}}^{\infty}\left[\frac{1}{f(\eta)} \int_{\eta}^{\infty} h(\zeta) d \zeta\right]^{1 / c} d \eta \leq \frac{w^{1-b / c}\left(\zeta\left(y_{2}\right)\right)}{b / c-1}<\infty .
$$

This contradicts ( $\mathrm{f}$ ) and proves the oscillation of all solutions.

The case where $w$ is an eventually negative solution is omitted since it can be dealt with similarly.

Next, we show that (f) is necessary. Assume that (f) does not hold, and let there exist $y \geq y_{0}$ such that:

$$
\int_{Y}^{y}\left[\frac{1}{f(\eta)}\left[\int_{\eta}^{\infty} h(\zeta) d \zeta\right]\right]^{1 / c} d \eta \leq \frac{1-a}{5},
$$

Let us consider the closed subset $M$ of continuous functions:

$$
M=\left\{w \in C\left(\left[y_{0}, \infty\right), \mathbb{R}\right): w(y)=\frac{1-a}{5}, y \in\left[y_{0}, Y\right] ; \frac{1-a}{5} \leq w(y) \leq 1 \text { for } y \geq Y\right\} .
$$

Then, we define the operator $\Phi: M \rightarrow C\left(\left[y_{0}, \infty\right), \mathbb{R}\right)$ by:

$$
(\Phi w)(y)=\left\{\begin{array}{l}
\frac{1-a}{5}, \quad y \in\left[y_{0}, Y\right] \\
-g(y) w(\vartheta(y))+\frac{1-a}{5}+\int_{Y}^{y}\left[\frac{1}{f(\eta)}\left[\int_{\eta}^{\infty} h(\zeta) f(w(\zeta(\zeta))) d \zeta\right]\right]^{1 / c} d \eta, \quad y \geq Y .
\end{array}\right.
$$

For every $x \in M$ and $y \geq Y,(\Phi w)(y) \geq \frac{1-a}{5}$ and:

$$
\begin{aligned}
(\Phi w)(y) & \leq a+\frac{1-a}{5}+\int_{Y}^{y}\left[\frac{1}{f(\eta)}\left[\int_{\eta}^{\infty} h(\zeta) d \zeta\right]\right]^{1 / c} d \eta \\
& \leq a+\frac{1-a}{5}+\frac{1-a}{5}=\frac{3 a+2}{5}<1
\end{aligned}
$$

which implies that $\Phi w \in M$. The rest of the proof follows from Theorem 1. The proof of the Theorem is complete. 
Example 2. Consider the delay differential equation:

$$
\left(\left(\left(w(y)-e^{-y} w(\vartheta(y))\right)^{\prime}\right)^{1 / 5}\right)^{\prime}+(y+1)(w(y-2))^{\frac{7}{3}}=0, \quad y \geq 0 .
$$

Here, $c=1 / 5, a=7 / 3, f(y)=1, \varsigma(y)=t-2, \Lambda(y)=y$. For $b=4 / 3$ and $p \leq q$, we have $a>b>c>0$ and $p^{a-b}=p \leq q^{a-b}=q$. To check $(f)$, we have:

$$
\int_{2}^{\infty}\left[\int_{\eta}^{\infty}(\zeta+1) d \zeta\right]^{5} d \eta=\infty
$$

Thus, all the assumptions of Theorem 3 hold. Hence, every solution of (12) oscillates or $\lim _{y \rightarrow \infty} w(y)=0$.

\section{Conclusions}

It is worth noting that the necessary and sufficient conditions we established hold when $-1<g(y) \leq 0$. These conditions do not hold in other ranges of $g(y)$. Therefore, the conditions we obtained hold in a limited range of $g(y)$.

At this point, we give one remark and two examples to conclude the paper.

Remark 2. The results in this paper also hold for equations of the form:

$$
\left.\left(f(y)\left((w(y)+g(y) w(\vartheta(y)))^{\prime}\right)^{c}\right)^{\prime}+\sum_{i=1}^{m} h_{i}(y) w^{a_{i}}\left(s_{i}(y)\right)\right)=0
$$

where $g, f, h_{i}, \varsigma_{i}(i=1,2, \ldots, m)$ satisfy Assumptions (a)-(d). Then, Theorems 1-3 can be extended, using an index $i$ such that $h_{i}, a_{i}, \varsigma_{i}$ fulfill (e) and $(f)$.

We conclude the paper by presenting two examples, which show how Remark 2 can be applied.

Example 3. Consider the delay differential equation:

$$
\left(e^{-y}\left(\left(w(y)-e^{-y} w(\vartheta(y))\right)^{\prime}\right)^{3 / 5}\right)^{\prime}+\frac{1}{y+1}(w(y-2))^{1 / 3}+\frac{1}{y+2}(w(y-1))^{1 / 5}=0, \quad y \geq 0 .
$$

Here, $c=3 / 5, a_{1}=1 / 3, a_{2}=1 / 5, f(y)=e^{-y}, g(y)=-e^{-y}, \varsigma_{1}(y)=y-2, \varsigma_{2}(y)=y-1$, $\Lambda(y)=\int_{0}^{y} e^{5 s / 3} d s=\frac{3}{5}\left(e^{5 y / 3}-1\right)$. For $b=1 / 2$ and $p \leq q$, we have $0<\max \left\{a_{1}, a_{2}\right\}<b<c$, and $p^{a_{1}-b}=p^{-1 / 6} \geq q^{a_{1}-b}=q^{-1 / 6}$ and $p^{a_{2}-b}=p^{-3 / 10} \geq q^{a_{2}-b}=q^{-3 / 10}$. To check (e), we have:

$$
\int_{0}^{\infty} \sum_{i=1}^{m} h_{i}(\eta) \Lambda^{a_{i}}\left(s_{i}(\eta)\right) d \eta \geq \int_{0}^{\infty} q_{1}(\eta) \Lambda^{a_{1}}\left(s_{1}(\eta)\right) d \eta=\int_{0}^{\infty} \frac{1}{\eta+1}\left(\frac{3}{5}\left(e^{5(\eta-2) / 3}-1\right)\right)^{1 / 3} d \eta=\infty
$$

since the integrand approaches $+\infty$ as $\eta \rightarrow+\infty$. Therefore, all the assumptions of Theorem 1 hold. Hence, every unbounded solution of (13) oscillates.

Example 4. Consider the delay differential equation:

$$
\left(\left(\left(w(y)-e^{-y} w(\vartheta(y))\right)^{\prime}\right)^{5 / 7}\right)^{\prime}+y(w(y-2))^{5 / 3}+(y+1)(w(y-1))^{3}=0, \quad y \geq 0
$$

Here, $c=5 / 7, a_{1}=5 / 3, a_{2}=3, f(y)=1, \varsigma_{1}(y)=y-2, \varsigma_{2}(y)=y-1, \Lambda(y)=y$. For $b=4 / 3$ and $p \leq q$, we have $\min \left\{a_{1}, a_{2}\right\}>b>c>0$ and $p^{a_{1}-b}=p^{1 / 3} \leq q^{a_{1}-b}=q^{1 / 3}$ and $p^{a_{2}-b}=p^{5 / 3} \leq$ $q^{a_{2}-b}=q^{5 / 3}$. Clearly, all the assumptions of Theorem 3 hold. Hence, every solution of (14) oscillates or $\lim _{y \rightarrow \infty} w(y)=0$. 
Author Contributions: Conceptualization, S.S.S., T.A.N., H.A. and O.B.; methodology, S.S.S., T.A.N., H.A. and O.B.; formal analysis, S.S.S., T.A.N., H.A. and O.B.; investigation, S.S.S., T.A.N., H.A. and O.B.; writing-original draft preparation, S.S.S., T.A.N., H.A. and O.B.; writing-review and editing, S.S.S., T.A.N., H.A. and O.B.; supervision, S.S.S., T.A.N., H.A. and O.B. All authors have read and agreed to the published version of the manuscript.

Funding: This research received no external funding.

Acknowledgments: The authors thank the reviewers for for their useful comments, which led to the improvement of the content of the paper.

Conflicts of Interest: The authors declare no conflict of interest.

\section{References}

1. Brands, J.J.M.S. Oscillation Theorems for second-order functional-differential equations. J. Math. Anal. Appl. 1978, 63, 54-64. [CrossRef]

2. Baculikova, B.; Dzurina, J. Oscillation Theorems for second-order neutral differential equations. Comput. Math. Appl. 2011, 61, 94-99. [CrossRef]

3. Baculikova, B.; Dzurina, J. Oscillation Theorems for second-order nonlinear neutral differential equations. Comput. Math. Appl. 2011, 62, 4472-4478. [CrossRef]

4. Bohner, M.; Grace, S.R.; Jadlovska, I. Oscillation criteria for second-order neutral delay differential equations. Electron. J. Qual. Theory Differ. Equ. 2017, 1-12. [CrossRef]

5. Chatzarakis, G.E.; Dzurina, J.; Jadlovska, I. New oscillation criteria for second-order half-linear advanced differential equations. Appl. Math. Comput. 2019, 347, 404-416. [CrossRef]

6. Chatzarakis, G.E.; Jadlovska, I. Improved oscillation results for second-order half-linear delay differential equations. Hacet. J. Math. Stat. 2019, 48, 170-179. [CrossRef]

7. Džurina, J. Oscillation Theorems for second-order advanced neutral differential equations. Tatra Mt. Math. Publ. 2011, 48, 61-71. [CrossRef]

8. Fisnarova, S.; Marik, R. Oscillation of neutral second-order half-linear differential equations without commutativity in delays. Math. Slovaca 2017, 67, 701-718. [CrossRef]

9. Umar, M.; Sabir, Z.; Raja, M.A.Z.; Shoaib, M.; Gupta, M.; Sanchez, Y.G. A Stochastic Intelligent Computing with Neuro-Evolution Heuristics for Nonlinear SITR System of Novel COVID-19 Dynamics. Symmetry 2020, 12, 1628. [CrossRef]

10. Guirao, J.L.G.; Sabir, Z.; Saeed, T. Design and numerical solutions of a novel third-order nonlinear Emden-Fowler delay differential model. Math. Probl. Eng. 2020, 2020, 7359242. [CrossRef]

11. Touchent, K.A.; Hammouch, Z.; Mekkaoui, T. A modified invariant subspace method for solving partial differential equations with non-singular kernel fractional derivatives. Appl. Math. Nonlinear Sci. 2020, 5, 35-48 [CrossRef]

12. Sabir, Z.; Raja, M.A.Z.; Guirao, J.L.G.; Shoaib, M. A Neuro-Swarming Intelligence-Based Computing for Second Order Singular Periodic Non-linear Boundary Value Problems. Front. Phys. 2020, 8, 224. [CrossRef]

13. Grace, S.R.; Džurina, J.; Jadlovska, I.; Li, T. An improved approach for studying oscillation of second-order neutral delay differential equations. J. Inequ. Appl. 2018, 193, 1-13. [CrossRef] [PubMed]

14. Karpuz, B.; Santra, S.S. Oscillation Theorems for second-order nonlinear delay differential equations of neutral type. Hacet. J. Math. Stat. 2019, 48, 633-643. [CrossRef]

15. Li, H.; Zhao, Y.; Han, Z. New oscillation criterion for Emden-Fowler type nonlinear neutral delay differential equations. J. Appl. Math. Comput. 2018, 60, 191-200. [CrossRef]

16. Agarwal, R.P.; Bohner, M.; Li, T.; Zhang, C. Oscillation of second-order differential equations with a sublinear neutral term. Carpathian J. Math. 2014, 30, 1-6.

17. Baculikova, B.; Li, T.; Dzurina, J. Oscillation Theorems for second order neutral differential equations. Electron. J. Qual. Theory Differ. Equ. 2011, 74, 1-13. [CrossRef]

18. Bazighifan, O.; Ruggieri, M.; Santra, S.S.; Scapellato, A. Qualitative Properties of Solutions of Second-Order Neutral Differential Equations. Symmetry 2020, 12, 1520. [CrossRef]

19. Li, T.; Rogovchenko, Y.V. Oscillation Theorems for second-order nonlinear neutral delay differential eqquations. Abstr. Appl. Anal. 2014, 594190.

20. Li, Q.; Wang, R.; Chen, F.; Li, T. Oscillation of second-order nonlinear delay differential equations with nonpositive neutral coefficients. Adv. Differ. Equ. 2015, 35, 1-7. [CrossRef] 
21. Pinelas, S.; Santra, S.S. Necessary and sufficient condition for oscillation of nonlinear neutral first-order differential equations with several delays. J. Fixed Point Theory Appl. 2018, 20, 1-13. [CrossRef]

22. Pinelas, S.; Santra, S.S. Necessary and sufficient conditions for oscillation of nonlinear first order forced differential equations with several delays of neutral type. Analysis 2019, 39, 97-105. [CrossRef]

23. Qian, Y.; Xu, R. Some new osciilation criteria for higher order quasi-linear neutral delay differential equations. Differ. Equ. Appl. 2011, 3, 323-335.

24. Santra, S.S. Existence of positive solution and new oscillation criteria for nonlinear first-order neutral delay differential equations. Differ. Equ. Appl. 2016, 8, 33-51. [CrossRef]

25. Santra, S.S. Oscillation analysis for nonlinear neutral differential equations of second-order with several delays. Mathematica 2017, 59, 111-123.

26. Santra, S.S. Oscillation analysis for nonlinear neutral differential equations of second-order with several delays and forcing term. Mathematica 2019, 61, 63-78. [CrossRef]

27. Santra, S.S. Necessary and sufficient condition for oscillatory and asymptotic behavior of second-order functional differential equations. Krag. J. Math. 2020, 44, 459-473. [CrossRef]

28. Papageorgiou, N.S.; Scapellato, A. Nonlinear Robin problems with general potential and crossing reaction. Rend. Lincei-Mat. Appl. 2019, 30,1-29. [CrossRef]

29. Papageorgiou, N.S.; Scapellato, A. Constant sign and nodal solutions for parametric $(p, 2)$-equations. Adv. Nonlinear Anal. 2020, 9, 449-478. [CrossRef]

30. Benia, Y.; Scapellato, A. Existence of solution to Korteweg-de Vries equation in a non-parabolic domain. Nonlinear Anal.-Theory Methods Appl. 2020, 195, 111758. [CrossRef]

31. Benia, Y.; Ruggieri, M.; Scapellato, A. Exact Solutions for a Modified Schrödinger Equation. Mathematics 2019, 7, 908. [CrossRef]

32. Ruggieri, M.; Speciale, M.P. Approximate Analysis of a Nonlinear Dissipative Model. Acta Appl. 2014, 132, 549-559. [CrossRef]

33. Hale, J. Theory of Functional Differential Equations. In Applied Mathematical Sciences, 2nd ed.; Springer: New York, NY, USA; Berlin/Heidelberg, Germany, 1977.

Publisher's Note: MDPI stays neutral with regard to jurisdictional claims in published maps and institutional affiliations.

(c) 2020 by the authors. Licensee MDPI, Basel, Switzerland. This article is an open access article distributed under the terms and conditions of the Creative Commons Attribution (CC BY) license (http:/ / creativecommons.org/licenses/by/4.0/). 